\title{
Candida krusei and Candida glabrata reduce the filamentation of Candida albicans by downregulating expression of HWP1 gene
}

\author{
Patrícia Pimentel de Barros $^{1} \cdot$ Fernanda Freire $^{1} \cdot$ Rodnei Dennis Rossoni $^{1}$ (D) \\ Juliana Campos Junqueira ${ }^{1}$ • Antonio Olavo Cardoso Jorge ${ }^{1}$
}

Received: 3 August 2016 / Accepted: 17 January 2017 / Published online: 6 February 2017

(C) Institute of Microbiology, Academy of Sciences of the Czech Republic, v.v.i. 2017

\begin{abstract}
Pathogenicity of Candida albicans is associated with its capacity switch from yeast-like to hyphal growth. The hyphal form is capable to penetrate the epithelial surfaces and to damage the host tissues. Therefore, many investigations have focused on mechanisms that control the morphological transitions of C. albicans. Recently, certain studies have showed that non-albicans Candida species can reduce the capacity of $C$. albicans to form biofilms and to develop candidiasis in animal models. Then, the objective of this study was to evaluate the effects of Candida krusei and Candida glabrata on the morphogenesis of $C$. albicans. Firstly, the capacity of reference and clinical strains of $C$. albicans in forming hyphae was tested in vitro. After that, the expression of HWP1 (hyphal wall protein 1) gene was determined by quantitative real-time PCR (polymerase chain reaction) assay. For both reference and clinical strains, a significant inhibition of the hyphae formation was observed when $C$. albicans was incubated in the presence of C. krusei or C. glabrata
\end{abstract}

Rodnei Dennis Rossoni

dennisrossoni@hotmail.com

Patrícia Pimentel de Barros

barrosdnapp@yahoo.com.br

Fernanda Freire

fefreire21@hotmail.com

Juliana Campos Junqueira

juliana@ fosjc.unesp.br

Antonio Olavo Cardoso Jorge

olavojorge@fosjc.unesp.br

1 Department of Biosciences and Oral Diagnosis, Institute of Science and Technology, Univ Estadual Paulista - UNESP, Francisco José Longo 777, São Dimas, São José dos Campos, SP CEP:12245-000, Brazil compared to the control group composed only by C. albicans. In addition, the culture mixed of C. albicansC. krusei or C. albicans-C. glabrata reduced significantly the expression of $H W P 1$ gene of C. albicans in relation to single cultures of this specie. In both filamentation and gene expression assays, $C$. krusei showed the higher inhibitory activity on the morphogenesis of C. albicans compared to C. glabrata. C. krusei and C. glabrata are capable to reduce the filamentation of $C$. albicans and consequently decrease the expression of the $H W P 1$ gene.

\section{Introduction}

A leading cause of opportunistic infections is fungal species belonging to the Candida genus, with $C$. albicans ranking as the most prevalent causative agent of candidiasis and candidemia around the world (Bassetti et al. 2015; Das et al. 2011; Guinea 2014; Nucci et al. 2013; Pfaller et al. 2012; Wisplinghoff et al. 2014). An important virulence factor of C. albicans is its ability to grow either as unicellular budding yeast or as filamentous forms, including hyphae and pseudohyphae (Fan et al. 2013; Lu et al. 2014; Shareck and Belhumeur 2011; Whiteway and Bachewich 2007). The hyphal form is responsible to promote tissue penetration and escape from immune cells leading to the infection process (Lu et al. 2014; Tati et al. 2016). The reversible morphological transition from yeast to hyphal filaments occur in response to a wide variety of conditions of the host environment that can be reproduced in in vitro studies, such as the presence of serum, body temperature $\left(37^{\circ} \mathrm{C}\right)$, high $\mathrm{CO}_{2}$ concentration, neutral $\mathrm{pH}$, certain carbon sources or amino acids, and extracellular matrix of microbial biofilms (Kadosh 2016).

The morphological switching of $C$. albicans is controlled by the protein products of specific genes. Among them, the 
hyphal wall protein 1 gene $(H W P 1)$ encodes a cell wall mannose protein that is essential for growth of hyphae (Fan et al. 2013). The HWP1 expression is induced by physical contact between the fungal and epithelial cells determining important functions for C. albicans, such as the binding of Candida to epithelial cells, the hyphal development, and the biofilm formation (Modrzewska and Kurnatowski 2015; Orsi et al. 2014).

Since the morphological transition is an important virulence mechanism for C. albicans, the factors evolved in this process constitute a target for the development of antifungal agents. A large number of small molecules such as farnesol, fatty acids, rapamycin, geldanamycin, histone deacetylase inhibitors, and cell cycle inhibitors have been reported to modulate the yeast-to-hypha transition in C. albicans (Shareck and Belhumeur 2011). Many of these molecules are produced as part of the quorum sensing (QS) that is a mechanism of microbial communication observed when different species are associated. The knowledge of the QS mechanisms whereby microorganisms compete with each other and establish antagonistic interactions may contribute to the discovery of new therapeutic strategies for human infections, such as C. albicans infections (Barbosa et al. 2016; Peleg et al. 2010).

In this context, some studies were developed to understand the mechanisms of interactions between Candida species. Thein et al. (2007a) showed that high concentrations of C. krusei were capable to suppress C. albicans populations in biofilms formed on acrylic surfaces. In previous studies, competitive interactions were also observed when C. albicans was associated with C. krusei and C. glabrata in mixed biofilms. Among these studies, Rossoni et al. (2015) verified that these competitive interactions attenuated the pathogenicity of $C$. albicans in animal models. Barros et al. (2016) observed that C. krusei and C. glabrata inhibited certain virulence genes of $C$. albicans. Moreover, dos Santos et al. (2016) found that non-albicans species were able to reduce the metabolic activity of $C$. albicans biofilms.

The vast majority of studies among Candida species were focused on biofilm assay and animal models, thus the present study evaluated the morphogenesis of $C$. albicans in the presence of non-albicans species. In this context, the objective was to investigate the effects of C. krusei and C. glabrata on C. albicans in relation to its ability to form hyphae in in vitro assays and to regulate the $H W P 1$ gene expression.

\section{Materials and methods}

\section{Candida strains}

The reference strains of C. albicans (ATCC18804), C. glabrata (ATCC90030), and C. krusei (ATCC6258) were obtained from the Oswaldo Cruz Institute, Rio de Janeiro, Brazil. The clinical strains were isolated from a single HIV- infected patient with oropharyngeal candidiasis lesions caused by a mixed infection of $C$. albicans (sample 60), C. krusei (sample 62), and C. glabrata (sample 64). The clinical isolates were collected at the Emílio Ribas Infectious Diseases Institute, São Paulo, Brazil, under the approval of the Ethics Committee (Protocol 051/2009-PH/CEP). The strains were stored in the Microbiology and Immunology Laboratory of the Institute of Science and Technology of São José dos Campos, UNESP-Univ. Estadual Paulista, São Paulo, Brazil.

\section{In vitro filamentation assay}

For this assay, the following groups were evaluated: C. albicans $+\operatorname{PBS}(n=5), C . k r u s e i+\operatorname{PBS}(n=5)$, C. glabrata + PBS $(n=5), C$. albicans $+C$. krusei $(n=5)$, and $C$. albicans $+C$. glabrata $(n=5)$. These groups were used for the references (total of $n=25$ ) and clinical strains (total of $n=25$ ). The experiment was performed independently in triplicate.

In a 24-well culture plate (Costar Corning, New York, NY, USA), $1 \mathrm{~mL}$ of distilled water was mixed with $10 \%$ fetal bovine serum and $50 \mu \mathrm{L}$ of the standardized C. albicans suspension $\left(10^{7}\right.$ viable cells per $\left.\mathrm{mL}\right)$. According to the experimental group, $50 \mu \mathrm{L}$ of standardized $C$. glabrata suspension $\left(10^{7}\right.$ viable cells per $\left.\mathrm{mL}\right)$ or $50 \mu \mathrm{L}$ of $C$. krusei $\left(10^{7}\right.$ viable cells per $\mathrm{mL}$ ) were also added. In the control groups, $50 \mu \mathrm{L}$ of PBS was added to each well. The plates were incubated at $37{ }^{\circ} \mathrm{C}$ under a partial pressure of $5 \% \mathrm{CO}_{2}$.

After $6 \mathrm{~h}$ of incubation, $50 \mu \mathrm{L}$ of the inoculum was transferred to glass slides with 10 previously demarcated fields on the back of the slide and observed under a light microscope at $\times 40$ magnification. The images were analyzed regarding C. albicans morphology and ten microscopic fields per slide were chosen for the quantification of hyphae. According to Ribeiro et al. (2016), the following scores were attributed for the number of hyphae present in each microscopic field: 0 , no hyphae; 1, 1-10 hyphae; 2, 11-20 hyphae; 3, 21-30 hyphae; 4, 31-40 hyphae; and 5, more than 41 hyphae. As C. krusei do not form true hyphae, only pseudohyphae in this assay both were quantified (total count of germination). The percentage of germination was calculated considering the heterotypic groups formed by $C$. albicans-C. glabrata or by C. albicans-C. krusei in relation to monotypic group of C. albicans.

\section{Quantitative real-time PCR assay}

The qReal-time PCR assay was performed in the same conditions described on in vitro filamentation assay. In this experiment, the control was the standardized suspension of C. albicans $\left(10^{7}\right.$ cells per $\left.\mathrm{mL}\right)$.

Total RNA of the groups described on in vitro filamentation assay was extracted using a TRIzol (Ambion, 
Inc., Carlsbad, CA, USA) as recommended by the manufacturer. The extracted total RNA (500 ng) was treated with DNase I (Turbo DNase Treatment and Removal ReagentsAmbion Inc., Carlsbad, CA, USA) and transcribed into complementary DNA (cDNA) using the SuperScript ${ }^{\circledR}$ III FirstStrand Synthesis SuperMix for qRT-PCR Kit (Invitrogen ${ }^{\mathrm{TM}}$, Carlsbad, CA, USA) according to the protocols recommended by the manufacturer.

The primer for $H W P 1$ gene was described according to Nailis et al. (2006, 2010). Its specificity was confirmed only for $C$. albicans and not for the other species of this genus (Barros et al. 2016). The transcribed cDNAs were amplified for relative quantification of the expression of the $H W P 1$ gene in relation to the concentration of the reference gene (RIPI).

The qPCR method was applied to evaluate the amount of the cDNA product in the exponential phase of the amplification reaction. As a detection system, the SYBR® Green fluorophore (Platinum ${ }^{\circledR}$ SYBR ${ }^{\circledR}$ Green qPCRSuperMixUDG Applied Biosystems, Framingham, MA, USA) was used as recommended by the manufacturer. As a negative control for the reaction, all of the reagents were added to the last wells of the plates except for cDNA, and the wells were sealed with optical adhesive (Invitrogen ${ }^{\mathrm{TM}}$, Carlsbad, CA, USA). Subsequently, the plate was placed in a StepOnePlus ${ }^{\mathrm{TM}}$ System (Applied Biosystems, Framingham, MA, USA) device, and the following cycling parameters were used: $50{ }^{\circ} \mathrm{C}$ for $2 \mathrm{~min}$, followed by an initial denaturation at $95{ }^{\circ} \mathrm{C}$ for $2 \mathrm{~min}$ and 40 cycles of $95^{\circ} \mathrm{C}$ for $15 \mathrm{~s}$ and $60{ }^{\circ} \mathrm{C}$ for $30 \mathrm{~s}$. The $2_{-\Delta \Delta \mathrm{CT}}$ method was used to analyze the relative changes in gene expression from the quantitative RT-PCR experiment (Livak and Schmittgen 2001).

\section{Statistical analysis}

Statistical analysis was performed using the GraphPad Prism5 software (GraphPad Software, Inc., La Jolla, CA, USA). The scores obtained by the analysis of in vitro filamentation were compared by the Kruskal-Wallis and Dunn's test. Student's $t$ test was used for relative quantification of gene expression assay. A $p$ value $\leq 0.05$ was considered significant.

\section{Results}

\section{Effects of $C$. glabrata and C. krusei on C. albicans filamentation}

In this assay, we observed a large number of $C$. albicans hyphae in the control groups with PBS. However, we verified a significant inhibition of the hyphae formation when C. albicans was incubated in the presence of C. krusei or C. glabrata compared to the control group. As expected, the control group of $C$. glabrata did not form hyphae. These results were obtained for both ATCC (Fig. 1a, b) and clinical strains (Fig. 2a, b).

The best reduction of $C$. albicans filamentation was observed in the interaction with $C$. krusei clinical strain, in which the median score of filamentation of $C$. albicans reduced significantly ( $p=0.0001)$ when this strain was cultivated with C. krusei. More specifically, the results obtained from C. albicans control was compared with heterotypic groups;
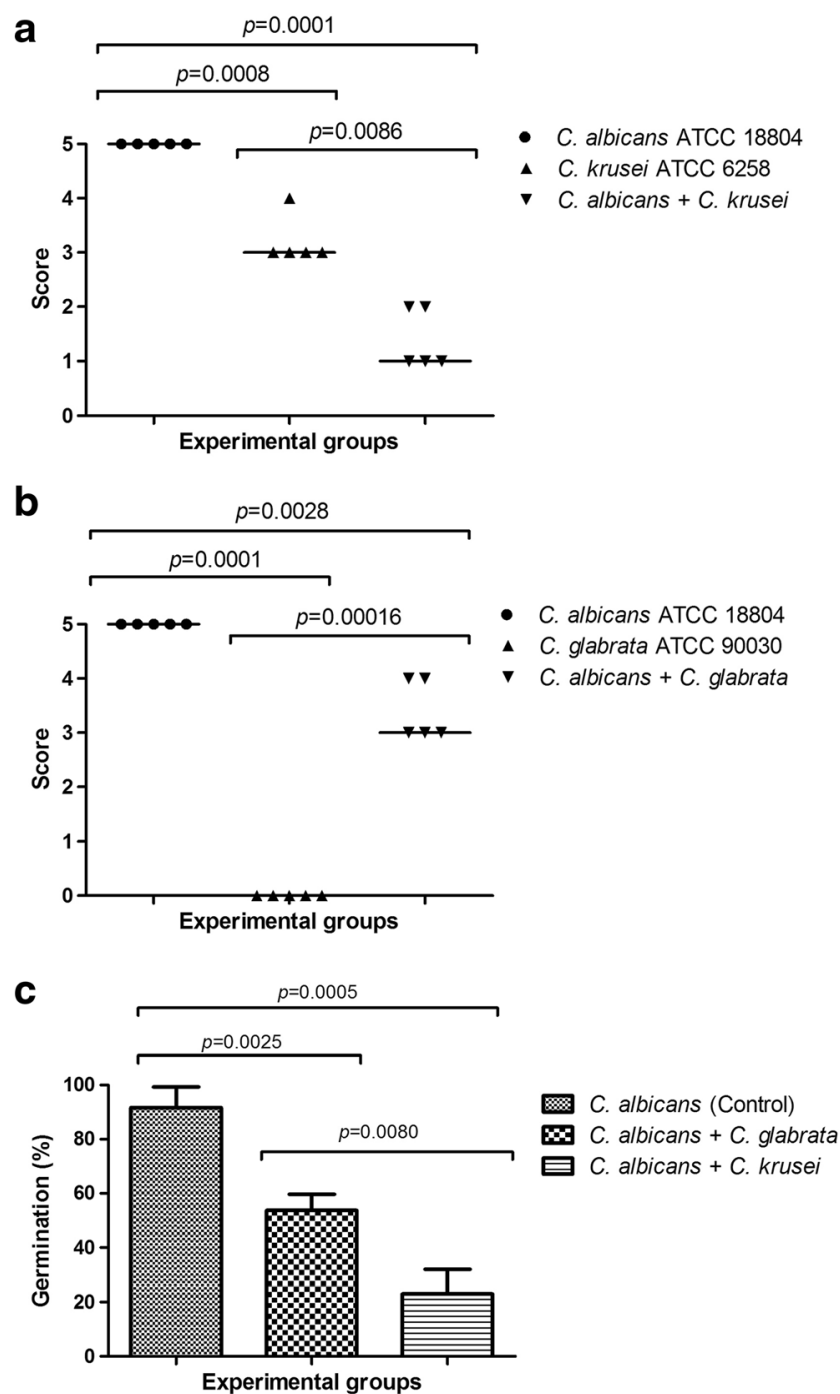

Fig. 1 Non-albicans Candida ATCC strains inhibit C. albicans filamentation. a Median scores obtained by determining the number of hyphae in the in vitro $C$. albicans filamentation assay for the following groups: C. albicans control group, C. krusei control group, and interaction group with $C$. krusei. b Median scores obtained by determining the number of hyphae in the in vitro $C$. albicans filamentation assay for the following groups: $C$. albicans control group, C. grabrata control group, and interaction group with C. glabrata. A significant hyphae reduction was observed in the interaction group when compared to the control groups (Mann-Whitney test, $p \leq 0.05$ ). c Percentage of germination, expressed as mean values of hyphae and pseudohyphae of $C$. albicans alone (control group) and when associated with $C$. glabrata and C. krusei ( $t$ test, $p \leq 0.05)$ 


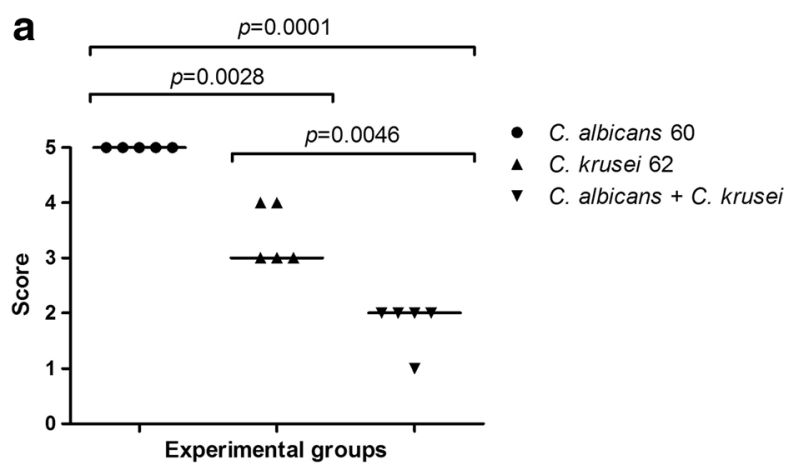

b
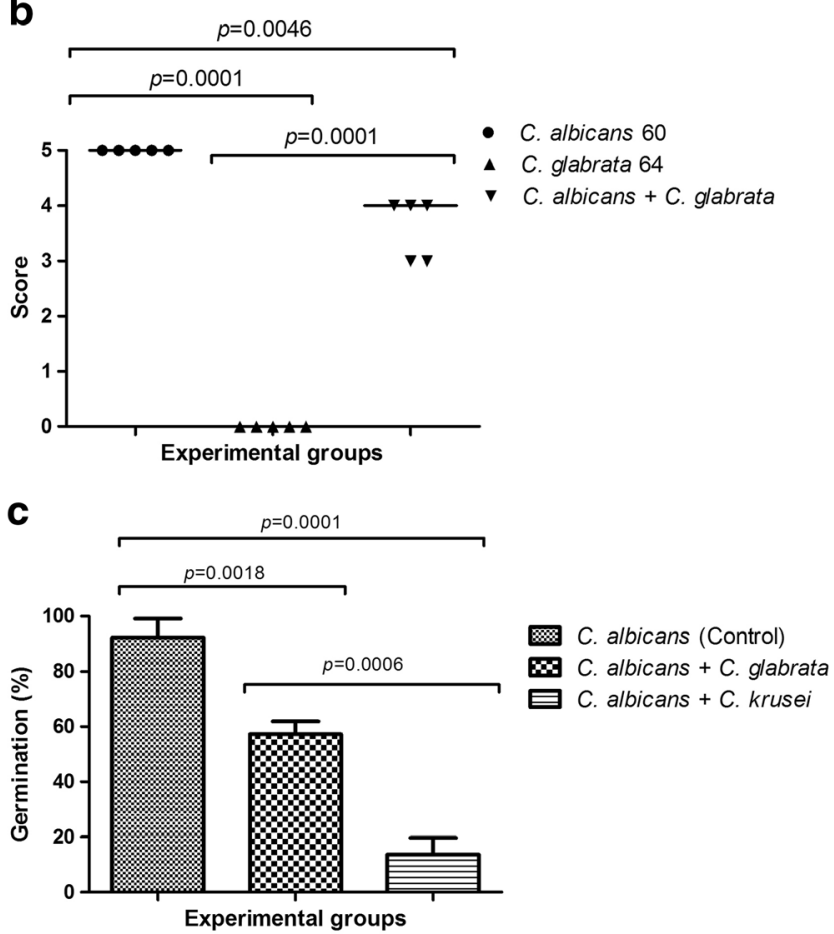

Fig. 2 Non-albicans Candida clinical strains inhibit C. albicans filamentation. a Median scores obtained by determining the number of hyphae in the in vitro $C$. albicans filamentation assay for the following groups: C. albicans control group, C. krusei control group, and interaction group with $C$. krusei. b Median scores obtained by determining the number of hyphae in the in vitro $C$. albicans filamentation assay for the following groups: C. albicans control group, C. grabrata control group, and interaction group with C. glabrata. A significant hyphae reduction was observed in the interaction group when compared to the control groups (Mann-Whitney test, $p \leq 0.05$ ). c Percentage of germination, expressed as mean values of hyphae and pseudohyphae of $C$. albicans alone (control group) and when associated with $C$. glabrata and $C$. krusei ( $t$ test, $p \leq 0.05)$

we found a $46.4 \%$ reduction in germination values when associated with C. glabrata (ATCC strain) opposed to $76.8 \%$ with C. krusei (ATCC strain) (Fig. 1c). For clinical strains, we found a reduction of $42.8 \%$ in germination values when associated with $C$. glabrata opposed to $86.2 \%$ with $C$. krusei (Fig. 2c), demonstrating that the inhibition of $C$. albicans was greater in the presence of $C$. krusei than in the presence of C. glabrata.

\section{Effects of $C$. krusei and $C$. glabrata on the expression of filamentation gene $H W P 1$ of $C$. albicans}

The expression levels of filamentation gene $H W P 1$ were quantified from cells of C. albicans ATCC 18804 and C. albicans 60 at the time of $6 \mathrm{~h}$ of the filamentation assay. C. albicans 60 strain exhibited a different gene expression pattern compared to the ATCC strain. The value of the transcript level of gene HWP1 in clinical strain was higher (3.89fold increase) than that observed in the reference strain (1.62fold increase) compared to C. albicans control (Fig. 3a, b).

In the interaction with $C$. krusei, for both reference and clinical strains, the HWP1 gene was downregulated with 20.25- and 15.56-fold decrease, respectively, showing significant differences compared with $C$. albicans after $6 \mathrm{~h}$ of filamentation assay $(p=0.0001)$. In the association with C. glabrata, both strains downregulated the HWP1 gene, with 4.37-fold decrease for reference and 6.48-fold decrease for clinical strains with statistical difference in relation to C. albicans after $6 \mathrm{~h}$ of filamentation assay $(p=0.0005)$ (Fig. 3a, b).

In agreement with the results obtained in the in vitro filamentation assays, the major inhibition of the HWP1 gene of $C$. albicans was observed in the presence of C. krusei.

\section{Discussion}

The ability of $C$. albicans to reversibly switch between yeast and filamentous forms is important for multiple aspects of biology and pathogenicity of this specie. C. albicans hyphal formation occurs in response to distinct environmental stimuli and is controlled by complex regulatory networks (Berman and Sudbery 2002; Brown and Gow 1999; Lopez-Ribot 2005). Since $C$. albicans and non-albicans Candida species are found together in candidiasis lesions (Junqueira et al. 2012; Samaranayake et al. 1987), in this study, we investigated the effects of C. krusei and C. glabrata on C. albicans in relation to its ability to form hyphae in in vitro assays and to regulate the $H W P 1$ gene expression.

Analysis of in vitro filamentation showed that the interaction between $C$. albicans and C. glabrata or C. krusei reduced the number of hyphae when compared to the control group inoculated only with $C$. albicans. The most reduction of C. albicans filamentation was observed in the interaction with C. krusei clinical strain, in which the median score of filamentation of $C$. albicans reduced $86.2 \%$ when this strain was cultivated with $C$. krusei. To the best of our knowledge, this is the first study that evaluated the influence of C. glabrata and C. krusei on the morphogenesis of C. albicans; consequently, there are no studies with similar methodology in order to compare and better discuss the present findings. 
Fig. 3 C. krusei and C. glabrata strains downregulated the expression of filamentation gene $H W P 1$ of $C$. albicans. a Relative quantification $(\log )$ of the expression of yeast-hyphae transition gene ( $H W P l)$ in filamentation assays after $6 \mathrm{~h}$ using quantitative real-time PCR (qPCR) in relation to the control in the ATCC strains. b Relative quantification $(\log )$ of the expression of yeast-hyphae transition gene (HWPl) in filamentation assays after $6 \mathrm{~h}$ using quantitative real-time PCR (qPCR) in relation to the control in the clinical strains. Values are expressed as the means and standard deviation. Student's $t$ test was used to compare gene expression $(p \leq 0.05)$. $* * *$ Indicates $p \leq 0.0001$

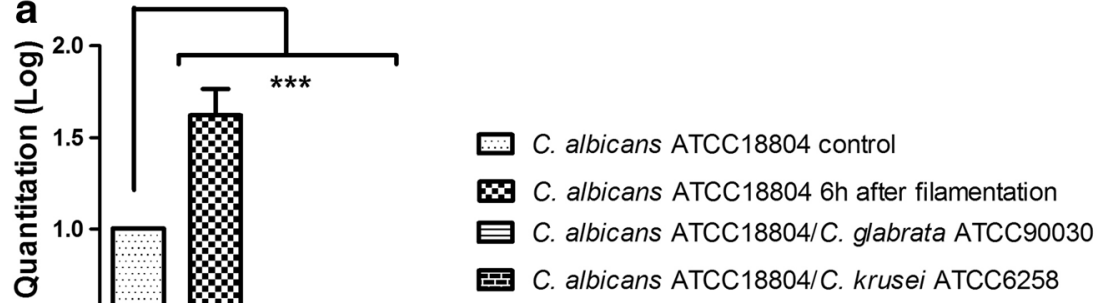

C. albicans 60 control

$\mathbf{B}$ C. albicans 60 th after filamentation

曰C. albicans 60/C. glabrata 64

臣 C. albicans 60/C. krusei 62
An important part of C. albicans pathogenicity is its ability to form hyphae, which plays important roles in the colonization and invasion of the host tissue. The hyphal form belongs to the overall factor of $C$. albicans virulence by invading epithelial and endothelial cells and evading phagocytes that cause the release of hydrolytic enzymes that lead to tissue damage (Tan et al. 2014). One hypothesis for our results is that the non-albicans Candida species can produce some signaling molecule that is capable to reduce $C$. albicans filamentation. In this context, the influence of $C$. krusei on C. albicans hyphae formation suggests new studies in order to elucidate the mechanisms of this interaction and may support new therapeutic protocols.

In the present study, we evaluated the expression of the C. albicans HWP1 gene in the filamentation assay when associated with non-albicans species. HWP1 gene is known to encode a major $C$. albicans protein involved in several functions, including cell wall assembly, intracellular signaling, and hyphal development; moreover, this gene promotes binding of Candida to epithelial cells, as the initial step of colonization (Biswas et al. 2007; Chaffin 2008; Naglik et al. 2006; Sundstrom et al. 2002). In the interaction with C. krusei and C. glabrata, the HWP1 gene was downregulated showing significant differences compared with control group. Several genes that have been identified to be expressed in a morphotype-dependent manner, "filamentspecific" genes and their products, have frequently been characterized as virulence factors such as ALS3 and HWP1 (Almeida et al. 2008; Phan et al. 2007).
In this study, the inhibitory influence of C. krusei and C. glabrata on the expression of the HWP1 gene of C. albicans agreed with Barros et al. (2016) that investigated the presence of these species on heterotypic biofilms at different times and may explain why Rossoni et al. (2015) found that single infections by $C$. albicans were more harmful for oral cavity of mice than mixed infections with non-albicans species.

Several hypotheses have been proposed to explain this intriguing phenomenon of inhibition between Candida species, such as competition for food and space, dissemination of inhibitory chemicals produced in the association and even the existence of mediators or molecular messengers that are able to reduce factors related to the virulence of $C$. albicans (Korres et al. 2011; Rossoni et al. 2015; Thein et al. 2007b). Furthermore, according to Thein et al. (2007b), the effect of C. krusei on C. albicans filamentation may be due to competitive adhesion in the early stages of biofilm formation, restricting populations of $C$. albicans and thereby the formation of hyphae.

In summary, we concluded that $C$. krusei and C. glabrata are capable to reduce the filamentation of C. albicans and consequently decrease the expression of the HWP1 gene. Our findings open new perspectives regarding molecular mechanisms involved in the ecological relationships between Candida species and these may assist to discover new alternative therapies for the control of candidiasis. 
Acknowledgements This study was supported by the São Paulo Council of Research-FAPESP, Brazil (Grants 2011/15194-0 and 2012/15250-0) for supporting this research.

Compliance with ethical standards The clinical isolates were collected at the Emílio Ribas Infectious Diseases Institute, São Paulo, Brazil, under the approval of the Ethics Committee (Protocol 051/2009-PH/ CEP).

Conflict of interest The authors declare that they have no conflict of interest.

\section{References}

Almeida RS et al (2008) The hyphal-associated adhesin and invasin Als3 of Candida albicans mediates iron acquisition from host ferritin. PLoS Pathog 4:e1000217. doi:10.1371/journal.ppat.1000217

Barbosa JO et al (2016) Streptococcus mutans can modulate biofilm formation and attenuate the virulence of Candida albicans. PLoS One 11:e0150457. doi:10.1371/journal.pone.0150457

Barros PP, Ribeiro FC, Rossoni RD, Junqueira JC, Jorge AO (2016) Influence of Candida krusei and Candida glabrata on Candida albicans gene expression in in vitro biofilms. Arch Oral Biol 64: 92-101. doi:10.1016/j.archoralbio.2016.01.005

Bassetti M et al (2015) Clinical and therapeutic aspects of candidemia: a five year single centre study. PLoS One 10:e127534. doi:10.1371/ journal.pone. 0127534

Berman J, Sudbery PE (2002) Candida albicans: a molecular revolution built on lessons from budding yeast. Nat Rev Genet 3:918-930. doi: $10.1038 / \mathrm{nrg} 948$

Biswas S, Van Dijck P, Datta A (2007) Environmental sensing and signal transduction pathways regulating morphopathogenic determinants of Candida albicans. Microbiology and molecular biology reviews: MMBR 71:348-376. doi:10.1128/MMBR.00009-06

Brown AJ, Gow NA (1999) Regulatory networks controlling Candida albicans morphogenesis. Trends Microbiol 7:333-338

Chaffin WL (2008) Candida albicans cell wall proteins. Microbiology and molecular biology reviews: MMBR 72:495-544. doi:10.1128/ mmbr.00032-07

Das I, Nightingale P, Patel M, Jumaa P (2011) Epidemiology, clinical characteristics, and outcome of candidemia: experience in a tertiary referral center in the UK. International journal of infectious diseases: IJID: official publication of the International Society for Infectious Diseases 15:e759-e763. doi:10.1016/j.ijid.2011.06.006

dos Santos JD, Piva E, Vilela SF, Jorge AO, Junqueira JC (2016) Mixed biofilms formed by $C$. albicans and non-albicans species: a study of microbial interactions. Braz Oral Res 30 doi:10.1590/18073107BOR-2016.vol30.0023

Fan Y, He H, Dong Y, Pan H (2013) Hyphae-specific genes HGC1, ALS3, HWP1, and ECE1 and relevant signaling pathways in Candida albicans. Mycopathologia 176:329-335. doi:10.1007/ s11046-013-9684-6

Guinea J (2014) Global trends in the distribution of Candida species causing candidemia. Clinical microbiology and infection: the official publication of the European Society of Clinical Microbiology and Infectious Diseases 20(Suppl 6):5-10. doi:10.1111/1469-0691. 12539

Junqueira JC et al (2012) Oral colonization by yeasts in HIV-positive patients in Brazil. Revista do Instituto de Medicina Tropical de Sao Paulo 54:17-24

Kadosh D (2016) Control of Candida albicans morphology and pathogenicity by post-transcriptional mechanisms. Cellular and molecular life sciences : CMLS. doi:10.1007/s00018-016-2294-y
Korres AM, Buss DS, Ventura JA, Fernandes PM (2011) Candida krusei and Kloeckera apis inhibit the causal agent of pineapple fusariosis, Fusarium guttiforme. Fungal biology 115:1251-1258. doi:10.1016/ j.funbio.2011.09.001

Livak KJ, Schmittgen TD (2001) Analysis of relative gene expression data using real-time quantitative PCR and the 2(-Delta Delta C(T)). Method Methods (San Diego, Calif) 25:402-408. doi:10.1006/ meth.2001.1262

Lopez-Ribot JL (2005) Candida albicans Biofilms: more than filamentation. Current biology : CB 15:R453-R455. doi:10.1016/j. cub.2005.06.020

Lu Y, Su C, Liu H (2014) Candida albicans hyphal initiation and elongation. Trends Microbiol 22:707-714. doi:10.1016/j.tim.2014.09. 001

Modrzewska B, Kurnatowski P (2015) Adherence of Candida sp. to host tissues and cells as one of its pathogenicity features. Annals of parasitology 61:3-9

Naglik JR, Fostira F, Ruprai J, Staab JF, Challacombe SJ, Sundstrom P (2006) Candida albicans HWP1 gene expression and host antibody responses in colonization and disease. J Med Microbiol 55:13231327. doi: $10.1099 / \mathrm{jmm} \cdot 0.46737-0$

Nailis H, Coenye T, Van Nieuwerburgh F, Deforce D, Nelis HJ (2006) Development and evaluation of different normalization strategies for gene expression studies in Candida albicans biofilms by real-time PCR. BMC Mol Biol 7:25. doi:10.1186/1471-2199-7-25

Nailis H, Kucharikova S, Ricicova M, Van Dijck P, Deforce D, Nelis H, Coenye T (2010) Real-time PCR expression profiling of genes encoding potential virulence factors in Candida albicans biofilms: identification of model-dependent and -independent gene expression. BMC Microbiol 10:114. doi:10.1186/1471-2180-10-114

Nucci M et al (2013) Epidemiology of candidemia in Latin America: a laboratory-based survey. PLoS One 8:e59373. doi:10.1371/journal. pone. 0059373

Orsi CF et al (2014) Impact of Candida albicans hyphal wall protein 1 (HWP1) genotype on biofilm production and fungal susceptibility to microglial cells. Microb Pathog 69-70:20-27. doi:10.1016/j. micpath.2014.03.003

Peleg AY, Hogan DA, Mylonakis E (2010) Medically important bacterialfungal interactions. Nat Rev Microbiol 8:340-349. doi:10.1038/ nrmicro2313

Pfaller M, Neofytos D, Diekema D, Azie N, Meier-Kriesche HU, Quan SP, Horn D (2012) Epidemiology and outcomes of candidemia in 3648 patients: data from the prospective antifungal therapy (PATH alliance(R)) registry, 2004-2008. Diagn Microbiol Infect Dis 74: 323-331. doi:10.1016/j.diagmicrobio.2012.10.003

Phan QT et al (2007) Als3 is a Candida albicans invasin that binds to cadherins and induces endocytosis by host cells. PLoS Biol 5:e64. doi:10.1371/journal.pbio.0050064

Ribeiro FC, de Barros PP, Rossoni RD, Junqueira JC, Jorge AO (2016) Lactobacillus rhamnosus inhibits Candida albicans virulence factors in vitro and modulates immune system in Galleria mellonella. Journal Applied of Microbiology. doi:10.1111/jam.13324

Rossoni RD et al (2015) Competitive interactions between C. albicans, C. glabrata and C. krusei during biofilm formation and development of experimental candidiasis. PLoS One 10:e131700. doi:10. 1371/journal.pone. 0131700

Samaranayake LP, MacFarlane TW, Williamson MI (1987) Comparison of Sabouraud dextrose and Pagano-Levin agar media for detection and isolation of yeasts from oral samples. J Clin Microbiol 25:162164

Shareck J, Belhumeur P (2011) Modulation of morphogenesis in Candida albicans by various small molecules. Eukaryotic cell 10:1004-1012. doi:10.1128/ec.05030-11

Sundstrom P, Balish E, Allen CM (2002) Essential role of the Candida albicans transglutaminase substrate, hyphal wall protein 1 , in lethal 
oroesophageal candidiasis in immunodeficient mice. The Journal of infectious diseases 185:521-530. doi:10.1086/338836

Tan X et al (2014) The role of Candida albicans SPT20in filamentation, biofilm formation and pathogenesis. PLoS One 9(4):e94468. doi:10. 1371/journal.pone.0094468

Tati S, Davidow P, McCall A, Hwang-Wong E, Rojas IG, Cormack B, Edgerton M (2016) Candida glabrata binding to Candida albicans hyphae enables its development in oropharyngeal candidiasis. PLoS Pathog 12:e1005522. doi:10.1371/journal.ppat.1005522

Thein ZM, Samaranayake YH, Samaranayake LP (2007a) Characteristics of dual species Candida biofilms on denture acrylic surfaces. Arch Oral Biol 52:1200-1208. doi:10.1016/j.archoralbio.2007.06.007
Thein ZM, Samaranayake YH, Samaranayake LP (2007b) In vitro biofilm formation of Candida albicans and non-albicans Candida species under dynamic and anaerobic conditions. Arch Oral Biol 52: 761-767. doi:10.1016/j.archoralbio.2007.01.009

Whiteway M, Bachewich C (2007) Morphogenesis in Candida albicans. Annu Rev Microbiol 61:529-553. doi:10.1146/annurev.micro.61. 080706.093341

Wisplinghoff $\mathrm{H}$ et al (2014) Nosocomial bloodstream infections due to Candida spp. in the USA: species distribution, clinical features and antifungal susceptibilities. Int J Antimicrob Agents 43:78-81. doi: 10.1016/j.ijantimicag.2013.09.005 